\title{
A Weibull Perspective on the Fracture of Atom Probe Specimens
}

\author{
Baptiste Gault ${ }^{1,2}$, Olivier Bouaziz ${ }^{3,4}$
}

1. Department of Materials Science \& Engineering, McMaster University, Hamilton, ON, Canada

2. now at Elsevier Ltd, Kidlington, UK

3. ArcelorMittal Research, Voie Romaine-BP30320, 57283, Maizières-lès-Metz Cedex, France

4. Centre des Matériaux/Mines Paris, Paristech, CNRS-UMR7633, BP 87, 91003, Evry cedex, France

Little is known and understood of the fundamental mechanisms underpinning the fracture of atom probe specimens during their analysis. Yet it is one of the main experimental limiting factors, as most experiments end with the specimen's fracture [1]. The stress associated with the electric field (electrostatic pressure) has been estimated via various methods [2-4] and is known to be very intense, in particular towards the specimen apex, where the electric field is enhanced by the lightning rod effect. Observation of elastic deformation [5] as well as stress-induced twinning [6] have both been reported.

The investigation by Moy et al. made use of finite-element simulations to derive the stress distribution along the specimen, and demonstrated a strong dependence on the specimen geometry. Interestingly, the fracture is known to occur somewhere down the shank of the specimen and not where the normal stress is the most intense, at the specimen apex. For a long time, this was only poorly understood. Here we propose that the Weibull theory (i.e. theory of weakest link) of brittle fracture can be applied to the fracture of atom probe specimens.

Atom probe experiments are performed at low temperature $(20 \mathrm{~K}-80 \mathrm{~K})$ we can consider that the fracture occurs in a brittle way. The probability of fracture is written as [7]: $\mathrm{P}\left(\sigma_{\mathrm{n}}, \mathrm{V}\right)=1-\exp \left(-\int_{\mathrm{o}}^{\mathrm{x}_{\mathrm{o}}}\left(\frac{\sigma_{\mathrm{n}}}{\sigma_{\mathrm{u}}}\right)^{\mathrm{m}} \cdot \frac{\mathrm{dV}}{\mathrm{V}_{\mathrm{o}}}\right)^{\mathrm{T}}$ where $\sigma_{\mathrm{u}}, \mathrm{V}_{\mathrm{o}}, \mathrm{m}$ are materials parameter and $\mathrm{dV}$ the volume under the normal stress $\sigma_{\mathrm{n}}$. The Weibull's parameters have been identified for some bulk materials (for tungsten $\sigma_{\mathrm{u}}=356 \mathrm{MPa}, \mathrm{m}=5.5, \mathrm{~V}_{\mathrm{o}}=1 \mathrm{~cm}^{3}$ [8]). Based on the results of Moy et al., we adapted the above formula to derive the fracture probability for an atom probe specimen, and as depicted in Figure 1. The approach seems to be relevant for predicting the locus of fracture, down along the specimen shank and not right at the tip of the specimen.

It is well documented that the outer-shape of atom probe specimens cannot be accurately described by a parabola, mostly due to a flattening of the apex during field evaporation. We hence derived a function $y=a \cdot\left(\frac{x}{x_{o}}\right)^{n} /\left(1+\frac{x}{x_{o}}\right)^{n-1}$ that allows reproducing the slow change in slope towards the apex, as well as the steadily increasing shank. The parameters $a$ and $x_{0}$ can be adjusted to reflect the radius of curvature and shank angle of the specimen. We used this function to fit experimental specimen shapes obtained by electron microscopy, as shown in Figure 2. We observed that using $n=3$ or 4 enabled to precisely fit the specimen shape. We finally extended the definition of the fracture probability to these functions and, as shown in Figure 3, the fracture probability is strongly dependent on the exact shape of the specimen for a similar shank angle and radius of curvature, which is in agreement with experimental observations.

References: 
[1] P.J. Birdseye, D.A. Smith, Surface Science, 23 (1970) 198-210.

[2] T.J. Wilkes et al., Journal of Physics D-Applied Physics, 5 (1972) 2226-2230.

[3] H.C. Eaton, R.J. Bayuzick, Surface Science, 70 (1978) 408-426.

[4] C.K.S. Moy, G. Ranzi, T.C. Petersen, S.P. Ringer, Ultramicroscopy, 111 (2011) 397-404.

[5] K.D. Rendulic, E.W. Müller, Journal of Applied Physics, 38 (1967) 2070-2072.

[6] K.D. Rendulic, E.W. Müller, Journal of Applied Physics, 37 (1966) 2593-2596.

[7] C.J. McMahon Jr, M. Cohen, Acta Metallurgica, 13 (1965) 591-604.

[8] J. Margetson, P.J. Sherwood, Journal of Materials Science, 14 (1979) 2575-2580.
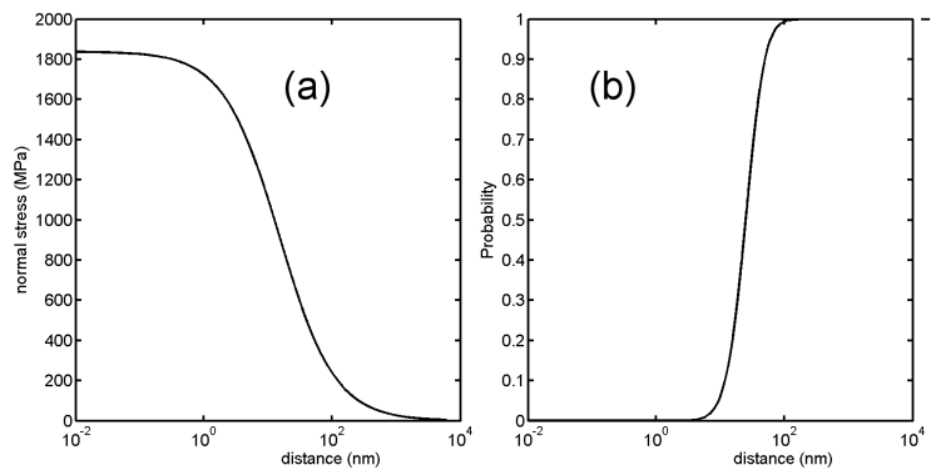

Figure 1. (a) Analytical expression derived from the numerical simulations from ref. [4], and (b) associated probability of brittle fracture as a function of the distance to the apex for a parabolic tip.
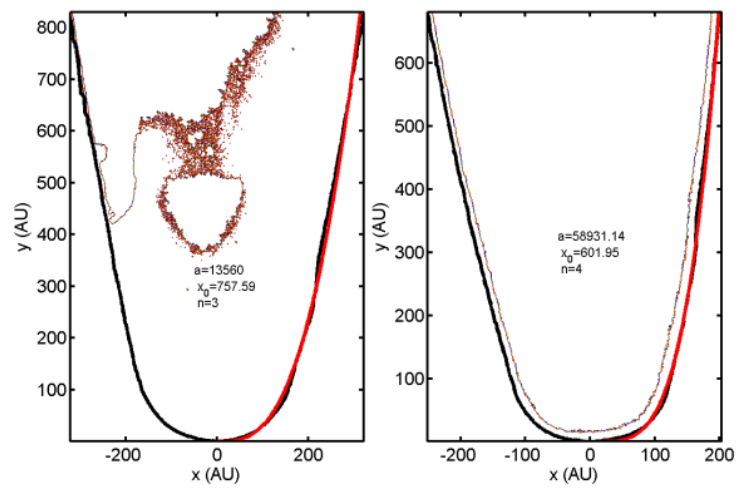

Figure 2. Extracted shape of pure tungsten atom probe specimens after analysis observed by electron microscopy and fitted function (red). The fitting parameters are inserted within the figure. Two examples are shown, one with a best fit obtained for $\mathrm{n}=3$ (left) and a second for $\mathrm{n}=4$ (right).

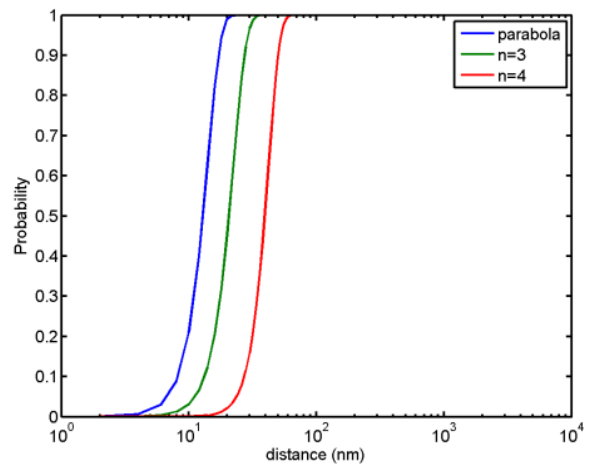

Figure 3. Brittle fracture probability as a function of the distance to the apex for three specimen shapes with similar shank angle and radius of curvature. 